\title{
Didactique et/ou didactiques? Des épistémologies et des postures, des approches et des méthodes
}

Didactique and/or Didactiques? Epistemologies, relationships, approaches and methods

\section{Francia Leutenegger}

\section{OpenEdition}

\section{Journals}

Édition électronique

URL : http://journals.openedition.org/educationdidactique/1889

DOI : 10.4000/educationdidactique.1889

ISSN : 2111-4838

Éditeur

Presses universitaires de Rennes

Édition imprimée

Date de publication : 30 juillet 2014

Pagination : $77-83$

ISSN : 1956-3485

Référence électronique

Francia Leutenegger, « Didactique et/ou didactiques ? Des épistémologies et des postures, des approches et des méthodes », Éducation et didactique [En ligne], 8-1 | 2014, mis en ligne le 15 septembre 2016, consulté le 02 mai 2019. URL : http://journals.openedition.org/ educationdidactique/1889; DOI : 10.4000/educationdidactique.1889 


\section{DIDACTIQUE ET/OU DIDACTIQUES ? DES ÉPISTÉMOLOGIES}

ET DES POSTURES, DES APPROCHES ET DES MÉTHODES 
La discussion portera sur les textes de Yves Reuter et de Jean-Louis Martinand. À partir de cette discussion critique, je prendrai position depuis le champ de la didactique comparée et plus particulièrement les travaux menés à Genève par notre équipe depuis le début des années 2000. Je reviendrai brièvement au fil du texte sur le projet initial de la didactique comparée en m'appuyant aussi sur les écrits de MariaLuisa Schubauer-Leoni, fondateurs de ce projet.

Afin de donner une certaine structure et unité au texte, l'option est de parcourir les termes du titre en cherchant à discuter les épistémologies et postures ainsi que les types d'approches et les méthodes mises en avant par chacun des deux auteurs. Ces différentes entrées seront mises en relation avec quelquesuns des thèmes de questionnements retenus par les organisateurs de la journée et traités par les auteurs. Après être revenue brièvement sur les questions de genre et de nombre (LA/LE/LES didactique(s)), je retiendrai trois espaces de questionnement : celui de la transmission culturelle, celui de la formation et celui de la recherche.

En exergue des différentes questions abordées, notons que les auteurs ont tous deux le mérite de tenter de faire de l'ordre dans les appellations, les désignations et les institutions concernées par LA/LE/ LES didactique(s), à savoir les institutions scolaire, académique, de recherche ou de formation qui ont toutes et chacune leur visée propre qu'il ne faut surtout pas confondre. Je me placerai surtout du point de vue de la recherche en didactique en cherchant à questionner les épistémologies et postures, dans les différents espaces concernés, avec une attention particulière à celle du chercheur en didactique ainsi que ses types d'approche et méthodes.

\section{DIDACTIQUE : DE GENRE ET DE NOMBRE?}

Lors de la journée d'étude, un premier étonnement de ma part a été de découvrir des positions assez radicales dans le débat sur LA didactique / LE didactique / LES didactiques alors même que le sens de ces termes me semblait relativement stabilisé depuis quelques années. Notons que ces questions de désignation ont aussi fait l'objet d'un débat nourri lors de l'édition récente de l'ouvrage "Didactique en construction, constructions des didactiques» (Dorier, Leutenegger \& Schneuwly, 2013, Éds.). Yves Reuter et Jean-Louis Martinand, quant à eux, se positionnent résolument pour un pluriel du terme « didactique » : les didactiques (des disciplines). Du reste, le syntagme complet, pour Yves Reuter, pose problème puisqu'il y voit un pléonasme : comment les didactiques peuvent-elles être autre chose que disciplinaires?

Il me semble que distinguer ces termes par un singulier ou un pluriel permet surtout de considérer le champ de recherche (LA didactique) commun à tout chercheur en didactique(s), des champs spécifiques aux objets disciplinaires qu'il représente. En effet le champ de la didactique est nécessairement pluriel (LES didactiques) puisqu'il prend la mesure des spécificités des objets d'enseignement/apprentissage. Mais que ce soit ce champ au sens large, par opposition à d'autres champs des sciences humaines et sociales - comme on nommerait celui de l'Histoire en regroupant du même coup les spécialités historiques de ses chercheurs sous une même bannière ou que cela concerne les disciplines d'enseignement spécifiques, toutes s'emploient à étudier ce qu'il en est de faits sociaux, ceux de la transmission des savoirs. Ces phénomènes sociaux relèvent, à mon sens, DU didactique, certes dense partout, comme le mentionnait Chevallard (1992) dans son anthropologie du didactique.

\section{DIDACTIQUE : UN MOUVEMENT DE TRANSMISSION CULTURELLE}

La discussion précédente amène, depuis le point de vue des deux auteurs, à interroger les postures épistémologiques relevant du questionnement sur le mouvement de transmission d'une « culture » qui est, nous disent les organisateurs de la journée, « à considérer au sens large, comme l'ensemble des savoirs, manières de faire et manières d'être qu'une société juge bon de transmettre aux générations nouvelles». La question est posée : « en quoi l'étude des conditions de diffusion/transmission de cette culture gagne-t-elle (ou non) à être structurée à l'image du découpage des savoirs, des pratiques, ou des activités au sein des institutions d'éducation et de formation? » (Ligozat, Coquidé \& Sensevy, dans ce numéro).

Poser la question DU didactique et celle d'une transmission culturelle aux jeunes générations revient aussi, depuis plusieurs années déjà, à postuler l'existence de phénomènes sociaux relevant de « quelque chose » de commun à ce qui s'enseigne et 
Francia Leutenegger

s'apprend, à l'école ou ailleurs, du reste. Les positions de Jean-Louis Martinand et Yves Reuter apparaissent ici relativement contrastées.

Pour le premier, on peut se sortir d'affaire en considérant que le point commun concerne le curriculum: il n'y a de didactiques (au pluriel) que les didactiques du curriculum puisque, - c'est particulièrement vrai au primaire, nous dit Jean-Louis Martinand- les différentes matières scolaires sont insérées dans un curriculum commun. Dès lors, les didacticiens (académiques) des disciplines sont porteurs «d'une responsabilité reconnue pour les contenus qu'elles partagent avec ce qu'on peut appeler les didactiques "praticiennes " d'enseignants et les didactiques "normatives " d'administrateurs..." (Martinand, dans ce numéro). Or cette question des curriculums n'est pas si simple et les frontières entre disciplines pas si "étanches » et Jean-Louis Martinand relève notamment la question des « éducations à...» qui font désormais partie du curriculum, sont par définition multidisciplinaires et posent des questions encore peu étudiées en didactique. Et de constater également qu' « il n'y a pas que des disciplines ou des matières à l'école, et les disciplines ne sont qu'une des formes, d'ailleurs très diversifiée que prend le curriculum scolaire..." (Martinand, dans ce numéro).

Je reprendrai cette discussion depuis mon point de vue de didacticienne comparatiste. Force est de constater que des « contenus de savoir» sont bien présents dans ces portions du curriculum dont parle Jean-Louis Martinand et sont donc du ressort des didacticiens. Mais quel(s) didacticien(s) sont en mesure d'étudier ces questions ? Un collectif de didacticiens (des disciplines scolaires) qui juxtaposerait différents points de vue " disciplinaires » ? Si oui, comment garantir la compatibilité épistémologique de ces points de vue ? Et quelle incidence sur les études didactiques subséquentes? Si le point commun est bien le curriculum, alors un travail plurididactique semble nécessaire pour étudier ces formes mouvantes du didactique à travers le curriculum, y compris des objets plus difficilement identifiables comme « disciplinaires ». Ces questions drainent le problème des modalités de ce travail commun mais surtout celui des appareils conceptuels mobilisés pour conduire ce type d'étude. Nous y reviendrons ci-dessous avec des questions plus méthodologiques.

Pour Yves Reuter, qui se fait aussi le porte-parole d'une équipe plus large, Théodile, il n'est de didactiques que disciplinaires et plurielles, et les notions de noyau dur et de configuration disciplinaire sont, semble-t-il, au premier abord, non reconductibles à des questions communes. Il rejoint également Jean-Louis Martinand sur la responsabilité des didacticiens quant aux contenus. Il distingue aussi, et cela lui semble fondamental, « des espaces différents d'actualisation des disciplines : un espace de prescriptions (par exemple, en France, les textes » officiels, (« un espace de recommandations (formation, inspection, associations, manuels, un espace de pratiques et un espace de représentations, ce qui permet, par voie de conséquence, de différencier des modes d'actualisation différents des disciplines : des configurations disciplinaires prescrites, recommandées, pratiquées) (re)construites par les acteurs. Donc, loin d'être essentialisées, les disciplines ont des modes d'existence différents » (Reuter, dans ce numéro).

Pour Yves Reuter, si les disciplines ont fondamentalement leur vie propre, quels sont alors l'intérêt et l'espace d'échanges possibles entre les didacticiens? Plusieurs pistes peuvent être esquissées sur la base des définitions apportées par l'auteur. Tout d'abord, il reconnaît que parmi les problèmes cruciaux, « celui des frontières initiale et finale des disciplines » (entre les disciplines scolaires), reste posé de même que celui de «l'entrée » et de la « sortie » d'une discipline : quand est-on, en tant qu'élève, « dans » une discipline et quand est-on « en dehors » de celle-ci, voire sur une transition entre les deux ? Mais alors comment se produit cette transition, de quoi est-elle faite? Et comment qualifier le « hors disciplinaire? » Celui-ci n'est-il « rien » ou est-il parfois (ou toujours ?) la marque de l'apprentissage d'une culture, en ce sens que l'apprentissage des contenus tire avec lui du «faire disciplinaire » (Reuter, dans ce numéro) ?

Je relèverai également trois points du texte d'Yves Reuter, que l'on pourrait, du reste, qualifier de communs aux différentes disciplines : le premier désigne les disciplines en tant que constructions sociales tandis que les deux autres, relevant, comme le premier, de la transposition, sont la forme scolaire prise par toutes les disciplines (Thévenaz-Christen 2008) et la fabrication de l'enseignable comme objectif fondamental de toute discipline (Chervel, 1988). Du point de vue de la recherche, comment dès lors étudier ces constructions sociales, ces formes scolaires et cette fabrication d'enseignables? Quel est l'intérêt de les étudier de manière commune entre didacticiens de différentes disciplines? La réponse d'Yves Reuter est claire : comparer. Comparer pour 
dégager les spécificités des disciplines, comparer pour faire émerger les points communs et les divergences d'un point de vue épistémologique. Mais cette réponse comparative ou comparatiste ${ }^{1}$, également présente dans le texte de Jean-Louis Martinand, ne résout pas la question des modalités de ce travail commun et celle des appareils conceptuels mobilisés : avec quels outils conceptuels, comparer ? Des outils propres à la didactique du français, à celle des mathématiques ou à quelle autre ? Ou bien ce type d'étude nécessite-t-il des outils conceptuels communs au regard des spécificités disciplinaires?

Si l'on considère la question du curriculum (selon Jean-Louis Martinand) ou la question des configurations disciplinaires (selon Yves Reuter), une question n'est pas abordée par les deux auteurs : qu'en est-il des temporalités dans ce curriculum et quelle est la place octroyée à certaines disciplines ? Quel espacetemps est consacré aux différentes disciplines, voire à ce qu'ils commentent comme les « éducations à... » ? Car si les objets ou contenus enseignés (pour reprendre la distinction que propose Yves Reuter) «formatent » sans doute les pratiques d'enseignement/apprentissage, qu'en est-il des choix (transpositifs) opérés sur ces objets en termes d'importance ou d'espace-temps ? Or, au moins à Genève ou ailleurs en Suisse, à petit espace-temps scolaire correspondent les dites "petites didactiques » qui correspondent également à un temps de formation plus restreint pour les futurs enseignants du primaire... D'un point de vue comparatiste, ces faits DU didactique (curriculaire) posent quelques questions et militent pour considérer, du point de vue de la recherche en didactique tout phénomène didactique au même titre: de ce point de vue il n'y a pas de « petites didactiques».

\section{DIDACTIQUE : UN ESPACE DE FORMATION}

Les questions de formation sont au cœur des textes des deux auteurs qui abordent, chacun à leur manière, le questionnement proposé par les organisateurs de la journée sur les «relations avec des dispositifs ou des institutions d'enseignement et de formation qui présentent une organisation de contenus d'apprentissage en termes de domaines d'étude ou de compétences » et les distinctions entre "didactique disciplinaire » et " approche didactique des processus d'enseignement et apprentissage " (Ligozat, Coquidé \& Sensevy, dans ce numéro).
À la suite de Jean-Paul Bronckart, pour qui « les didactiques sont avant tout des sciences de l'ingénierie culturelle » (citée par Jean-Louis Martinand, dans ce numéro), Jean-Louis Martinand note deux orientations de recherche possibles en didactique : une orientation (en tant que science fondamentale ?) vers des travaux visant la compréhension des phénomènes d'enseignement et d'apprentissage et une orientation en tant que sciences de l'ingénierie culturelle. Cette position, somme toute assez proche des visées de Guy Brousseau et de sa théorie des situations didactiques en mathématiques (Brousseau 1998), assortie des méthodes d'ingénierie, conduit à une réflexion sur le rôle des didactiques dans la formation des enseignants qui, pour l'auteur, est fondamental. Jean-Louis Martinand souligne toutefois " l'incommensurabilité entre pratiques de recherche et pratiques d'enseignement ». Il est alors essentiel de problématiser les questionnements en vue de la formation. Je rejoins volontiers cette dernière position : si les didacticiens sont indispensables à la formation des enseignants, ils sont toutefois peu ou mal préparés à ce que j’appellerai, moi, une forme de transposition des objets issus de la recherche en didactique dans le cadre de dispositifs de formation à l'enseignement. Ils ne le sont pas plus à l'égard de dispositifs de formation à la recherche, puisque les didacticiens interviennent non seulement dans la formation à l'enseignement, mais aussi dans la formation à la recherche en didactique. Ces questions devraient, à mon sens, faire l'objet de développements futurs de la didactique, en tant que « didactique de la didactique ».À noter à cet égard la parution toute récente d'un ouvrage consacré à ces questions, depuis la didactique comparée et sur la base de textes de différents auteurs (Leutenegger, Amade-Escot \& Schubauer-Leoni (Éds.), 2014).

Une autre question d'importance est abordée par les deux auteurs : celle de la formation spécifique pour les futurs enseignants du primaire, qui sont des enseignants généralistes, par différence avec ceux du secondaire. Jean-Louis Martinand s'interroge sur cette spécificité au regard du fait qu'en France les futurs professeurs des écoles sont recrutés au niveau master et sont donc déjà formés dans une matière particulière. En Suisse, la question se pose différemment puisque les futurs enseignants primaires (la plupart d'entre eux) commencent leur formation (d'une durée de quatre ans) à l'issue du diplôme de maturité (baccalauréat). Ils reçoivent 
Francia Leutenegger

ainsi une formation en sciences de l'éducation qui comprend différentes approches, notamment celles des didactiques. Quelle est alors l'articulation entre les différentes disciplines didactiques participant à cette formation? Pour les praticiens du primaire, comment générer une réflexivité au-delà des différentes postures épistémologiques véhiculées par les contenus de formation rencontrés dans les différents modules de didactiques des disciplines ? Postures dont les ressources pour l'enseignement se font volontiers l'écho (voir à ce sujet, Schubauer-Leoni \& Leutenegger, 2009). La question serait de savoir s'il est opportun, utile, voire nécessaire que l'enseignant primaire soit au fait des différences à ce niveau-là et, si la réponse est oui, qu'il soit en mesure d'en tirer les conséquences pour son enseignement.

\section{DIDACTIQUE : UN ESPACE DE RECHERCHE}

Ces caractéristiques épistémologiques drainent aussi la question des relations entre didactiques et sciences de l'éducation et Jean-Louis Martinand, comme Yves Reuter, explorent le double ancrage du didacticien dans sa discipline d'origine et en sciences de l'éducation. Cette question est également au cour des préoccupations de Maria-Luisa Schubauer-Leoni lorsqu'elle préconise une "entrée par les savoirs » alliée à " une entrée par les sujets », pour le développement des recherches en didactique (SchubauerLeoni, 1998/2001). Cette position épistémologique me semble fondamentale pour la recherche en didactique comparée parce qu'elle est la seule qui permette une véritable cohérence avec le parti pris systémique des didacticiens (la fameuse triade enseignant-élèvessavoir). Par ailleurs, la construction d'une théorie de l'action conjointe en didactique (Sensevy $\&$ Mercier, 2007 (Éds.) trouve sa justification dans cette double entrée justement. Enfin, et pour revenir à l'articulation entre didactiques et sciences de l'éducation, elle est probablement une bonne candidate à un dialogue avec des approches plus transversales chères aux sciences de l'éducation, mais surtout elle semble indispensable à la formation des enseignants (qui sont en sciences de l'éducation). En effet, comment se passer d'une entrée par les sujets des systèmes didactiques, dès lors que ces futurs professionnels sont censés se former aux gestes de la profession?

Les questions précédentes nous ramènent aux questions posées par les organisateurs de la journée :
«Au-delà des conditions spécifiques de transmission et diffusion de contenus dans les processus d'enseignement et apprentissage, peut-on dégager de nouveaux modes de structuration possibles des recherches en didactiques? Jusqu'à quel point cette structuration est-elle dépendante de la spécificité des systèmes éducatifs auxquels ces recherches sont adossées ? " (Ligozat, Coquidé \& Sensevy, dans ce numéro). Sur ce point, je réagis à certaines prises de positions des deux auteurs à ce sujet.

Pour introduire le sujet, je pars d'une distinction qui, pour être évidente, n'est toutefois pas si facile à prendre en compte par le chercheur en didactique du fait de son insertion dans la formation et son implication, parfois, dans les curriculums. Je distingue les différentes disciplines propres à ce curriculum - moyennant les avertissements de Jean-Louis Martinand et d'Yves Reuter concernant les objets multidisciplinaires - avec leurs spécificités en tant qu'objets appartenant au terrain scolaire et l'étude que peut réaliser la recherche en didactique sur ces objets. Confondre discipline et didactique disciplinaire (ou didactique tout court) ne reviendrait-il pas à confondre l'objet avec l'instrument pour étudier l'objet ? Car si je peux suivre Yves Reuter sur le fait que les différentes disciplines, avec leur « faire didactique », leur épistémologie propre et leur « configuration disciplinaire » sont à examiner dans leurs spécificités, je ne peux le suivre sur la dérive qu'il mentionne: "Cette dernière dérive possible signale d'ailleurs l'illusion selon laquelle un unique chercheur pourrait être spécialiste de toutes les disciplines, de tous leurs contenus, de toute l'histoire de toutes les didactiques et interroge la création de postes de didactique comparée, stratégie potentiellement dangereuse à mon sens en ce qu'elle peut entrainer le dommage irrémédiable de la perte du travail en commun de spécialistes des différentes disciplines, perspective particulièrement stimulante justement ouverte par le projet comparatiste » (Reuter, dans ce numéro). Je ne crois pas avoir déjà vu mentionné qu'un chercheur en didactique comparée devait se charger d'un type d'étude recouvrant toutes les disciplines et toute l'histoire de toutes les didactiques... Qui pourrait prétendre à une chose pareille? D'une part, un domaine de recherche en général (et pas seulement en didactique) n'est jamais une affaire individuelle; il se construit dans un collectif regroupant des chercheurs qui partagent un certain nombre de questions et s'appuient sur des épistémologies 
communes pour les traiter. D'autre part, le principe d'un collectif est de réunir des compétences spécifiques permettant d'alimenter plusieurs axes de travail au sein du domaine de recherche. Ainsi, le didacticien comparatiste est doté d'une spécialisation disciplinaire plus ou moins étendue selon son parcours personnel, mais quoi qu'il en soit, l'exercice du comparatisme l'amène nécessairement à se faire «spécialiste " opportun d'objets ou contenus de savoir configurés dans d'autres discipline(s) dans le cadre de collaborations avec d'autres chercheurs. Ces collaborations sont motivées par le traitement d'une question commune, qui en général ne recoupe pas exactement les préoccupations des chercheurs dits «spécialistes» des disciplines concernées. Cela signifie, entre autres que le tenant d'une chaire de didactique comparée a pour qualité première de ne pas craindre de se confronter à l'altérité disciplinaire. Faut-il redouter de voir se répandre un exercice intellectuel nouveau ? Plus raisonnablement, je préfère penser que la crainte, inspirée par le développement de chaires de didactique comparée, est somme toute assez banale dans un monde académique, où toute forme de nouveauté fait redouter un déclin des courants existants, ne serait-ce que par une redistribution des ressources. Le cœur du problème devient alors le suivant : la création de chaires de didactique comparée est-elle synonyme d'une certaine hégémonie d'un domaine didactique sur les autres? Selon moi, cette suspicion est infondée et, dans ce qui suit, j'en donne quelques principales raisons.

Il faut commencer par lever une confusion souvent réitérée, à propos de l'appellation de l'Association pour des Recherches Comparatistes en Didactique (ARCD). Pour rappel, l'association n'est pas une association de "didactique comparée " comme le sous-entend Yves Reuter lorsqu'il dit que « ce projet - celui de la didactique comparée - se développe difficilement, à voir le faible nombre de membres de notre Association, et au risque de trois dérives (...)» (Reuter, dans ce numéro). L'association, dont font partie des membres d'équipes de didactique comparée au même titre que des didacticiens des disciplines, résulte d'un projet collectif justement, et probablement bien plus large que ceux dont les équipes de didactique comparée sont en mesure de se charger. Quant au faible nombre de membres (environ une centaine de chercheurs), celui-ci n'est pas significativement plus faible que celui de certaines associations de didactiques disciplinaires, compte tenu de leur ancienneté et de leur capacité à drainer des chercheurs sous l'angle d'une discipline charriant les valeurs que le système scolaire et la société lui attribue. Curieusement, il existe deux " puissantes » associations de didacticiens du français et des mathématiques respectivement, mais qu'en est-il des associations de didacticiens de l'histoire, de la musique, ou de la technologie?

De mon point de vue, l'existence de chaires de didactique comparée est intimement liée également à des projets collectifs où différents spécialistes de disciplines œuvrent ensemble, au sein d'une même équipe, avec la particularité de se doter de cadres conceptuels plus unifiés pour étudier LE didactique, et mieux à même d'établir les comparaisons chères à Yves Reuter. Des cadres conceptuels permettant de saisir et de décrire des phénomènes didactiques issus de différentes pratiques didactiques (des « faire didactiques » probablement), de différentes institutions, de différents terrains. À noter également que ces études comparatistes ne sont pas nécessairement interdisciplinaires (Mercier, Schubauer-Leoni \& Sensevy, 2002, Éds.) : d'autres axes comparatistes ont été esquissés et travaillés tels que les transitions entre les formes institutionnelles (crèche lécole; primaire /secondaire) à propos d'un même objet de savoir ou encore la comparaison des formes de pratiques didactiques liées à une discipline, dans différents contextes nationaux ou internationaux. Que ces études se situent au plan interdisciplinaire, interinstitutionnel, international ou autres, il ne s'agit en aucun cas d'une « vision surplombante», comme le suppose Yves Reuter, mais plutôt d'études de type « bottom up » prenant leurs objets dans les différentes réalités didactiques qu'elles explorent pour en faire émerger les spécificités et les généricités, c'est du moins le projet initial de la didactique comparée.

Dès lors, si le point de vue est pluriel (comparatiste), cela signifie que ce pluriel doit s'organiser. De telles problématiques didactiques, portées par des spécialistes des disciplines concernées et donc par des équipes et non des individus, nécessitent de se doter des instruments conceptuels et méthodologiques permettant la comparaison entre les différents espaces didactiques qui, justement, parce qu'ils ont leurs spécificités, doivent être reconduits à des observables semblables ou tout au moins comparables. Je rejoins du reste Jean-Louis Martinand qui, à ce sujet, parle d'une "spécificité des problématiques didactiques ».Celles-ci s'étendent à des problématiques 
Francia Leutenegger

prenant en compte différents espaces didactiques peu explorés de manière conjointe.

Je reprendrai aussi les termes d'Yves Reuter qui distingue "les visées proprement disciplinaires des visées qui sont communes aux différentes disciplines et propres à l'école et celles qui excèdent le cadre scolaire: en d'autres termes, les disciplines visent à construire un sujet disciplinaire, un sujet scolaire et un sujet social »(Reuter, dans ce numéro). Si les disciplines visent à la fois ces trois constructions, qu'en est-il des didactiques (disciplinaires ?) en tant que science(s) ? Cette science plurielle peut-elle se passer d'une réflexion sur l'articulation entre les trois pôles du système ? Qui se charge de cette étude et comment ? Et comment se doter des outils conceptuels et méthodologiques permettant de traiter ces comparaisons "au-delà des faux rapprochements immédiats » dont parle Jean-Louis Martinand. Une première réponse donnée par la didactique comparée consiste à construire des concepts visant une meilleure connaissance des processus didactiques dans des espaces distincts ou conjoints grâce aux sujets didactiques, à les mettre à l'épreuve de l'observation et de l'analyse et, cas échéant, à leur apporter de meilleures adaptations. Ces tentatives sont nouvelles et demandent une grande prudence, alliée à une bonne dose de modestie permettant de préserver une nécessaire vigilance épistémique.

\section{NOTES}

1. Voir à cet égard la distinction proposée par TutiauxGuillon (2013) : « il y a un écart entre faire une recherche comparative en didactique " et "faire une recherche comparatiste "; la première compare des données parce que c'est une méthodologie classique en sciences sociales, alors que la seconde prend pour objet la comparaison entre disciplines, entre didactiques, entre concepts dans une approche didactique. Du coup les savoirs produits non seulement éclairent les disciplines scolaires, mais contribuent aussi à l'épistémologie des didactiques. » (p. 263).

\section{RÉFÉRENCES}

Brousseau, G. (1998). La théorie des situations didactiques en mathématiques. Grenoble : La Pensée Sauvage éditions

Chevallard, Y. (1992). Concepts fondamentaux de la didactique : perspectives apportées par une approche anthropologique. Recherches en didactique des mathématiques, vol. 12/1, 73-112.

Chervel, A. (1998). La culture scolaire. Une approche historique. Paris : Belin.

Dorier, J.-L., Leutenegger, F. \& Schneuwly, B. (Éds.) (2013). Didactique en construction, constructions des didactiques. Collection Raisons Éducatives. Paris, Bruxelles : De Boeck.

Leutenegger, F., Amade-Escot, C. \& Schubauer-Leoni, M.L. (Éd.) (sous presse). Interactions entre recherches en didactique(s) et formation des enseignants. Questions de didactique comparée. Presses Universitaires de FrancheComté.

Mercier, A., Schubauer-Leoni, M.-L., \& Sensevy, G. (Éd.). (2002). Vers une didactique comparée. Revue Française de pédagogie, 141.

Schubauer-Leoni, M.-L. (1998/2001). Les sciences didactiques parmi les sciences de l'éducation : l'étude du projet scientifique de la didactique des mathématiques. In R. Hofstetter \& B. Schneuwly (Éd.), Le pari des sciences de l'éducation (p. 329-352). Paris, Bruxelles : De Boeck Université. Coll. Raisons Éducatives.

Schubauer-Leoni, M.-L. \& Leutenegger, F. (2009). Implicites dans l'étude des processus transpositifs. Quelques repères dans les manuels scolaires de mathématiques et de français des premières années de scolarité. In C. Cohen-Azria \& N. Sayac (Éd.) Questionner l'implicite. Les méthodes de recherche en didactiques (3) (p. 243-259. Villeneuve d'Ascq : Presses universitaires du Septentrion.

Sensevy, G. \& Mercier, A. (Éds.) (2007). Agir ensemble. Laction didactique conjointe du professeur et des élèves. Presses Universitaires de Rennes.

Thevenaz-Christen, T. (2008). Au cœur de la forme scolaire, la discipline. L'exemple du français parlé. Revue suisse des Sciences de l'éducation, 2, 299-324.

Tutiaux-Guillon, N. (2013). Comparer les didactiques? In B. Daunay, Y. Reuter, \& A. Thépaut (Éds.), Les contenus disciplinaires. Approches comparatistes (p. 263-273). Lille : Presses Universitaires du Septentrion. 